\title{
Uso de frutos artificiais associados à cameras trap no estudo de frugivoria
}

\section{Use of artificial fruits associated with cameras trap in the study of frugivory}

\author{
Adriano José Nepomuceno Amorim ${ }^{1 *}$, Eriki Miller Lima Luiz Paiva ${ }^{1}$, Camila Aoki ${ }^{1,2}$
}

\begin{abstract}
RESUMO
Frutos artificiais têm sido utilizados para testar várias hipóteses ecológicas, entre elas a preferência de cores por frugívoros. Contudo, entre as desvantagens já apresentadas para este método, estava a impossibilidade de identificação das espécies envolvidas na interação. O objetivo deste estudo foi conciliar o uso de frutos artificiais com o de cameras trap. O estudo foi realizado na Fazenda Novo Horizonte, no Pantanal de Aquidauana. Foram utilizados 120 frutos artificiais, divididos igualmente em três cores (vermelho, preto e verde). Foram registradas duas espécies de aves com a utilização de cameras trap, Choca-do-planalto (Thamnophilus pelzelni, Thamnophilidae) e o Sabiá-barranco (Turdus leucomelas, Turdidae) e os frutos selecionados foram os de cor vermelha e preta. O número de registros foi baixo, porém a associação de cameras trap à estudos envolvendo frutos artificiais permitiu a identificação das aves, possibilitou a observação de preferência específica de cores e também a descrição dos comportamentos durante a interação.
\end{abstract}

Palavras-chave: Armadilhas fotográficas; Frugívoros; Thamnophilus pelzelni; Turdus leucomelas.

\begin{abstract}
Artificial fruits have been used to test several ecological hypotheses, including the color preference of frugivores. However, among the disadvantages already presented for this method was the impossibility of identifying the species involved in the interaction. The aim of this study was to reconcile the use of artificial fruits with that of camera traps. The study was carried out at Fazenda Novo Horizonte, in the Pantanal de Aquidauana. 120 artificial fruits were used, divided equally into three colors (red, black and green). Two species of birds were recorded with the use of cameras traps, the Planalto Slaty Antshrike (Thamnophilus pelzelni, Thamnophilidae) and the Pale-breasted Thrush (Turdus leucomelas, Turdidae) and the selected fruits were red and black. The number of records was low, but the association of cameras traps to studies involving artificial fruits allowed the identification of birds, allowed the observation of specific color preference and also the description of behavior during the interaction.
\end{abstract}

Keywords: Camera traps; Frugivores; Thamnophilus pelzelni; Turdus leucomelas.

\footnotetext{
${ }^{1}$ Universidade Federal de Mato Grosso do Sul, Campus Universitário de Aquidauana - CPAQ. CEP 79200-000 - Aquidauana, MS, Brasil.

*E-mail: adrianonepomuceno22@ hotmail.com

${ }^{2}$ Programa de Pós-Graduação em Recursos Naturais (FAENG) e Programa de Pós-Graduação em Biologia Vegetal (INBIO), Universidade Federal de Mato Grosso do Sul, CEP 79070-900 Campo Grande, MS, Brasil.
} 


\section{INTRODUÇÃO}

A polpa e os tecidos comestíveis de frutos são componentes fundamentais na dieta das aves, suplementando a alimentação e contribuindo na dispersão de sementes (JORDANO, 2000). Aves frugívoras são aquelas que utilizam frutos como parte da dieta, sendo classificadas entre espécies generalistas ou especialistas (SNOW, 1981). Aves especialistas são aquelas que se alimentam preferencialmente de frutos e que, via de regra, abandonam as sementes em condições de germinação (MCKEY, 1975; SNOW, 1981). Aves generalistas ou oportunistas satisfazem suas necessidades nutricionais por meio de fontes animais e vegetais, o que promove diferentes níveis de danos às sementes (MCKEY, 1975). A eficiência de dispersão dessas sementes é, portanto, variável entre as espécies de frugívoros (HOWE; ESTABROOK, 1977).

As técnicas de forrageio dos frugívoros variam sazonalmente, respondendo a estímulos de oferta e de procura e, de acordo com essa demanda, a maneira de forrageio das aves é guiada pelas necessidades fisiológicas (CORLETT, 2011). Por serem visualmente orientadas seu forrageio geralmente inclui frutos coloridos (JANSON, 1983) e com sinais de maturescência (MOERMOND; DENSLOW, 1983).

Determinar os fatores que influenciam a escolha dos frutos pelas aves pode ajudar na compreensão das interações entre plantas e seus dispersores (LEVEY et al., 1984). A utilização de frutos artificiais funciona como alternativa para manipular independentemente as variáveis envolvidas no consumo de frutos por aves, buscando-se assim encontrar padrões nesses consumos. Com esta técnica, pode-se controlar o número de frutas, a sua posição e, até certo ponto, a sua cor e tamanho (ALVES-COSTA \& LOPES 2001). Contudo, são apontadas como desvantagens desta técnica, os danos que os frutos sofrem por conta da ação de insetos, sua curta atratividade e a dificuldade de saber se a remoção foi causada por aves, mamíferos ou outros fatores (ALVES-COSTA \& LOPES, 2001).

Neste sentido, propomos nesse trabalho a conciliação do uso de frutos artificiais com o de cameras trap, de modo a permitir a identificação dos animais que realizam o consumo dos frutos e a descrição dos seus comportamentos.

\section{MATERIAL E MÉTODOS}


Este trabalho foi conduzido entre outubro de dezembro de 2017 e 2018, na Fazenda Novo Horizonte (20¹3'36”S, 55 51'49'W), localizada no Pantanal, sub-região de Aquidauana (MS). O Pantanal brasileiro situa-se entre os paralelos $16^{\circ}$ e $21^{\circ} \mathrm{S}$ e os meridianos $55^{\circ}$ e $58^{\circ} \mathrm{W}$, com altitudes variando de 100 a $150 \mathrm{~m}$ (MERCANTE et al., 2011). O clima apresenta sazonalidade bem definida, temperatura média de cerca de $25^{\circ} \mathrm{C}$ e precipitação média anual de cerca de $1.400 \mathrm{~mm}$, variando entre 800 e 1.600 mm (ANA, 2005). O período de chuvas, que vai de outubro a abril (ROHLI \& VEGA, 2008) concentra cerca de 70 a $80 \%$ da média pluviométrica anual.

Para este experimento, foram utilizados 120 frutos artificiais, divididos igualmente em três cores: preto, vermelho e verde (formato esférico, com diâmetro de 2 $\mathrm{cm}$ ). Os frutos artificiais foram confeccionados com massa de modelar (plastilina, Acrilex $\left.{ }^{\circledR}\right)$ à base de amido, atóxica, sem odor e resistente à água (conforme ALVESCOSTA \& LOPES, 2001). As três cores foram distribuídas em conjuntos de cinco, em árvores sem flores ou frutos selecionados na vegetação nativa (altura média de 1,70m).

Após a fixação dos frutos nas árvores, foram instaladas cameras trap Bushnell próximas destes. A coleta de dados foi realizada nos meses de outubro e novembro de 2017. Os frutos artificiais permaneceram em exposição por sete dias consecutivos, em média. Foram 840 horas de amostragem com câmera trap, as gravações tiveram 60 segundos de duração, iniciando a partir da captação de movimento pelo sensor da câmera, configurado em alta sensibilidade, com intervalos de 10 segundos para cada filmagem. A permanência da ave em filmagens subsequentes foi contabilizada como um único registro.

Foram anotadas as espécies de animais envolvidas, o horário, número de indivíduos de cada espécie de animal, a cor de fruto selecionada e modo de coleta e ingestão de frutos (FITZPATRICK, 1980). Os animais foram identificados através de guias de campo (SIGRIST, 2007; SOUZA, 2002; PIVATTO; BERNARDON; ENDRIGO, 2012) e a nomenclatura segue o proposto pelo CBRO (2014).

\section{RESULTADOS E DISCUSSÃO}

Apenas duas espécies de aves foram registradas nas cameras trap. Choca-doplanalto (Thamnophilus pelzelni, Thamnophilidae, Figura 1A) foi registrada às $08: 18 \mathrm{~h}$, selecionou os frutos vermelhos e exibiu o comportamento descrito por Fitzpatrick (1980) como apanhar empoleirado, no qual as aves pousam no ramo com frutos e comem ali 
mesmo. Esta espécie é classificada como insetívora, habita beira de matas, inclusive secundárias (SIGRIST, 2006).

O sabiá-barranco (Turdus leucomelas, Turdidae, Figura 1B), às 09:44h apanhou empoleirado (FITZPATRICK, 1980) tanto frutos pretos quanto frutos vermelhos durante o evento de frugivoria. Turdus leucomelas esteve entre as espécies com maior número de interações de frugivoria em plantas nesta mesma área em estudo desenvolvido por Arguelho (2017). Esta é uma espécie semi-florestal de vasta distribuição, considerada onívora e se alimenta principalmente de frutos e invertebrados (SICK, 2001; SIGRIST, 2006).

Figura 1 - (A) Thamnophilus pelzelni e (B) Turdus leucomelas, registrados em eventos de frugivoria no Pantanal de Aquidauana, MS
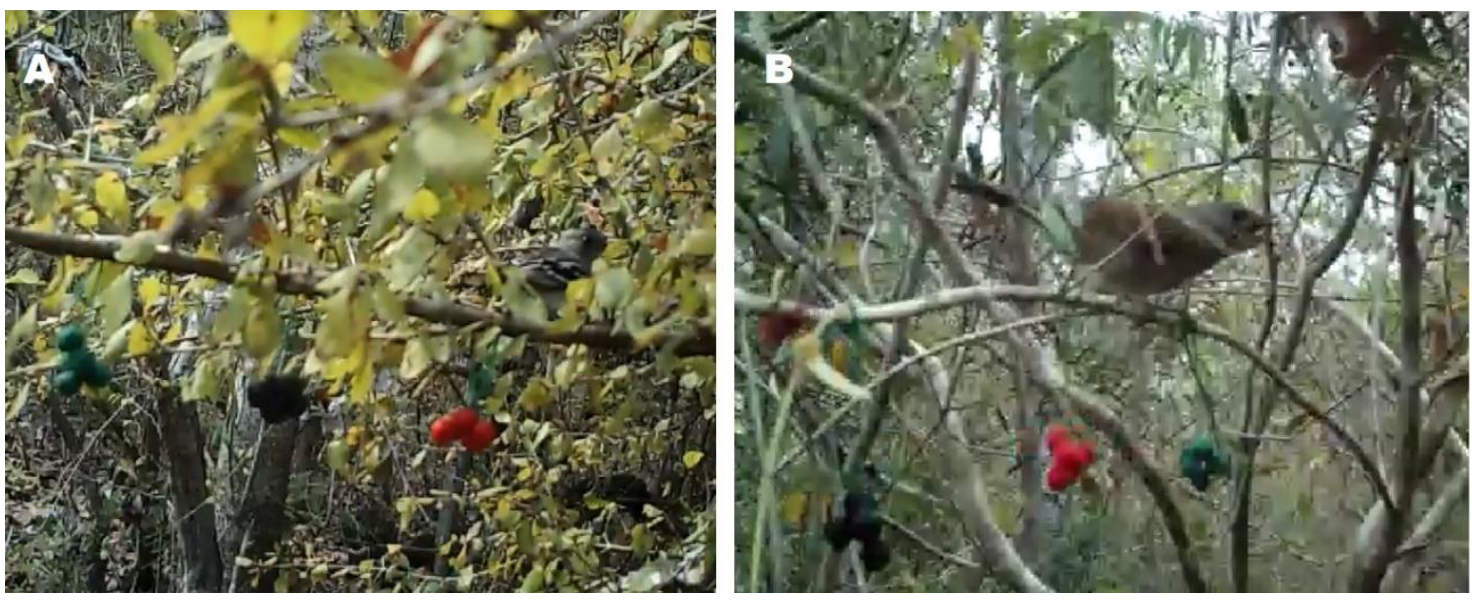

Fonte: Amorim (2017) - em cameras trap Bushnell

Apesar do elevado número de horas de camera trap (840h) o número de registros de frugivoria foi baixo, o que não nos permite tecer comentários a respeito da preferência por cores de frutos, embora frutos verdes não tenham sido consumidos. $\mathrm{O}$ uso de frutos artificiais na natureza é muito eficiente, pois permitem testar hipóteses e compreender o comportamento dos frugívoros dentro do processo ecológico (ALVES-COSTA \& LOPES, 2001; GALETTI; ALVES-COSTA; GAZETTA, 2003).

Entre as desvantagens citadas para o uso dos frutos artificiais está a dificuldade de saber qual o frugívoro envolvido na interação (ALVES-COSTA \& LOPES, 2001) e a impossibilidade de identificação de preferências de cada espécie, sendo comum apenas a descrição de um padrão geral para a comunidade (ALVES-COSTA; LOPES, 2001; ARRUDA; RODRIGUES; IZZO, 2008; AGUIAR; BISPO, 2013). 
Cameras trap vem atualmente sendo utilizadas para o registro de frugívoros (JAYASEKARA et al., 2007; O'BRIEN \& KINNAIRD, 2008; PRASAD et al., 2010; EATON et al., 2016) e tem a vantagem de permanecer por longos períodos (inclusive noturnos), não afugentar os animais e possibilitar ao pesquisador assistir repetidas vezes os vídeos, que pode fazer com que detalhes de comportamento possam ser observados e descritos. Contudo apresenta desvantagem de ser um método caro e a área coberta ser relativamente pequena, se comparada com a área observada pelo pesquisador.

A associação de cameras trap à estudos envolvendo frutos artificiais nos permitiu identificar não apenas os autores da frugivoria, mas também os horários de forrageio das espécies, seus comportamentos e preferências pela cor do fruto. Sendo assim, indicamos que estudos com amostragem maior sejam realizados para complementar essas informações.

\section{AGRADECIMENTOS}

O presente trabalho foi realizado com apoio da Coordenação de Aperfeiçoamento de Pessoal de Nível Superior - Brasil (CAPES) - Código de Financiamento 001. Agradecemos à Universidade Federal de Mato Grosso do Sul - UFMS/MEC - Brasil e à Fundação de Apoio ao Desenvolvimento do Ensino, Ciência e Tecnologia do Estado de Mato Grosso do Sul (FUNDECT - Processo n59/300.048/2015) pelo apoio aos projetos desenvolvidos pelo grupo de pesquisa "Estudos Integrados em Biodiversidade do Cerrado e Pantanal".

\section{REFERÊNCIAS}

AGUIAR, A. G.; BISPO, A. A. Efeitos da coloração e da distribuição de frutos artificiais nas taxas de consumo por aves em um fragmento florestal. Bioikos, v. 27, p. 57-65, 2013.

ALVES-COSTA, C. P.; LOPES, A. V. F. Using artificial fruits to evaluate fruit selection by birds in the field. Biotropica, v. 33, p. 713-7, 2001.

ANA. Strategic action program of the integrated Management of the Pantanal and the upper Paraguay River Basin - Final Report. Agência Nacional das Águas, 2005. 
Arguelho, W. C. Redes de interações entre plantas e aves frugívoras no Pantanal Sul-mato-grossense. Trabalho de Conclusão de Curso. UFMS, Campus Aquidauana, 2017.

ARRUDA, R.; RODRIGUES, D.J.; IZZO, T.J. Rapid assessment of fruit-color selection by birds using artificial fruits at local scale in Central Amazonia. Acta Amazonica, v 38, p. 291-296, 2008.

BURNS, K. C; DALEN, J. L. Foliage color constrasts and adaptive fruit color variation in a bird-dispersed plant community. Oikos, v. 96, p. 463-69, 2002.

CORLETT, R.T. How to be a frugivore (in a changing world). Acta Oecologica, v. 37, p. $674-81,2011$.

EATON, D.P.; KEUROGHLIAN, A.; SANTOS, M.C.A.; DESBIEZ, A.L.J.; SADA, D.W. Citizen scientists help unravel the nature of cattle impacts on native mammals and birds visiting fruiting trees in Brazil's southern Pantanal. Biological Conservation. v. 208, p. 29-39, 2016.

FITZPATRICK, J. W. 1980. Foraging behavior of neotropical tyrant flycatchers. Condor, v. 82, p. 43-57, 1980.

GALETTI, M, ALVES-COSTA, C.P.; GAZETTA, E. 2003. Effects of forest fragmentation, anthropogenic edges and fruit colour on the consumption of ornithocoric fruits. Biological Conservation, 111:269-73.

HART, N.S. The visual ecology of avian photoreceptors. Progress in Retinal and Eye Research, p. 675-703, 2001.

HOWE, H.F.; ESTABROOK, G.F. On intraspecific competition for avian dispersers in tropical trees. The American Naturalist, v. 111, p. 817-32, 1977.

JANSON, C.H. Adaptation of fruit morphology to dispersal agentes in a Neotropical forest. Science, v. 219, p. 187-9, 1983.

JAYASEKARA, P.; WEERASINGHE, U.R.; WIJESUNDARA, S.; TAKATSUKI, S. Identifying diurnal and nocturnal frugivores in the terrestrial and arboreal layers of a tropical rain forest in Sri Lanka. Ecotropica, v. 13, p. 7-15, 2007.

JORDANO, P. Patterns of mutualistic interactions in pollination and seed dispersal: connectance, dependence asymmetries, and coevolution, p. 657-677. The University of Chigago Press. 1987.

JORDANO, P. 2000. Fruits and frugivory, p. 125-66. In: FENNER M. (ed.) Seeds: The ecology of regeneration in plant communities. Wallingford (UK). CABI Publ.

LEVEY, D. J.; MOEMOND T. C.; DENSLOW, J. S. Fruit choice in neotropical birds: The effect of distance between fruits on preferences patterns. Ecology, v. 65, p. 844-50, 1984. 
MCKEY, D. The ecology of coevolved seed dispersal systems, p. 159-91. In: L.E. GILBERT, L. E.; RAVEN, P. (ed.) Coevolution of animals and plants. Austin (TX). Texas Press. 1975.

MOEMOND, T.C.; DENSLOW, J.S. Fruit choice in Neotropical birds: Effects of fruit type and acessibility on selectivity. Journal of Animal Ecology, v. 52, p. 407-20, 1983.

O'BRIEN, T. G.; KINNAIRD, M.F. A picture is worth a thousand words the application of camera trapping to the study of birds. Bird Conservation International, v. 18, p. 144-162, 2008.

PIVATTO, M. A. C.; BERNARDON, G.; ENDRIGO, E. Guia fotográfico Aves do Pantanal. São Paulo (SP). Aves \& Fotos Editora. 2012.

PRASAD, S.; PITTET, A.; SUKUMAR, R. Who really ate the fruit? A novel approach to camera trapping for quantifying frugivory by ruminants. Ecological Research, v. 25, p. 225-231, 2010.

ROHLI, R. V.; VEJA, A. J. Climatology. Boston (MA). Jones and Bartlett Publishers. 2008.

SICK, H. Ornitologia brasileira. Rio de janeiro. Ed. Nova Fronteira, 2000.

SIGRIST, T. Aves do Brasil: uma visão artística. São Paulo. Fosfertil, 2006.

SIGRIST, T. Guia de Campo: Aves do Brasil Oriental. São Paulo (SP). Editora Saraiva, 2007.

SNOW, D.W. 1981. Tropical frugivorous birds and their food plants: A word survey. Biotropica, v. 13, p. 1-14.

SOUZA, D. All the birds of Brazil: an Identification Guide. Salvador (BA). Editora Dall. 2002.

\section{Recebido em: 10/112021}

Aprovado em: 12/12/2021

Publicado em: 17/12/2021 